\title{
Multi-time-over-threshold technique for photomultiplier signal processing: Description and characterization of the SCOTT ASIC
}

\author{
S. Ferry ${ }^{a}$, F. Guilloux ${ }^{b}, *$, S. Anvar ${ }^{b}$, F. Chateau ${ }^{b}$, E. Delagnes ${ }^{b}$, V. Gautard ${ }^{b}$, F. Louis ${ }^{b}$, E. Monmarthe ${ }^{b}$, \\ H. Le Provost ${ }^{b}$, S. Russo ${ }^{c}$, J.-P. Schuller ${ }^{a}$, Th. Stolarczyk ${ }^{a}$, B. Vallage ${ }^{a}$, E. Zonca $^{b}$ \\ ${ }^{\text {a } C E A / I r f u / S P P, ~ G i f-s u r-Y v e t t e, ~ F r a n c e ~}$ \\ ${ }^{\mathrm{b}}$ CEA/Irfu/SEDI, Gif-sur-Yvette, France \\ ${ }^{\mathrm{c}}$ Dipartimento di Scienze Fisiche Università di Napoli, Napoli, Italy
}

\section{Representing the KM3NeT Consortium}

\section{A R T I C L E I N F O}

Available online 15 October 2011

\section{Keywords:}

KM3NeT

Neutrino detection

PMT

Time-over-threshold

ASIC

\begin{abstract}
A B S T R A C T
KM3NeT aims to build a cubic-kilometer scale neutrino telescope in the Mediterranean Sea based on a 3D array of photomultiplier tubes. A dedicated ASIC, named SCOTT, has been developed for the readout electronics of the PMTs: it uses up to 16 adjustable thresholds to digitize the signals with the multitime-over-threshold technique. Digital outputs of discriminators feed a circular sampling memory and a "first in first out" digital memory for derandomization. At the end of the data processing, the ASIC produces a digital waveform sampled at $800 \mathrm{MHz}$. A specific study was carried out to process PMT data and has showed that five specifically chosen thresholds are suited to reach the required timing precision. A dedicated method based on the duration of the signal over a given threshold allows an equivalent timing precision at any charge. A charge estimator using the information from the thresholds allows a charge determination within less than $20 \%$ up to 60 pe.
\end{abstract}

(c) 2011 Elsevier B.V. All rights reserved.

\section{Introduction}

High energy cosmic rays have been discovered almost a century ago, yet their origin remains unknown. For the study of their origins, electrically neutral particles are necessary as they are not deflected by interstellar magnetic fields. Neutrinos at $\mathrm{TeV}$ energies are expected to be produced in large fluxes as a byproduct of cosmic ray interaction in cosmic accelerators, via the decay of pions. Since neutrinos interact weakly, they can escape dense regions where they are generated and act as direct messengers. On the other hand a large volume must be instrumented, of the order of a cubic kilometer, to reach an adequate sensitivity.

The KM3NeT [1] consortium is in the preparatory phase of such a neutrino telescope in the Mediterranean Sea. In the TeV region and above, a charged current interaction of a muon neutrino produces a muon almost collinear to the incoming neutrino. The Cherenkov light emitted by the muon crossing the detector is detected by a 3-dimensional array of photomultiplier tubes (PMT). The amount of light is proportional to the muon

\footnotetext{
* Corresponding author.

E-mail address: fabrice.guilloux@cea.fr (F. Guilloux).
}

energy above $1 \mathrm{TeV}$. The time and amplitude of the signal allow reconstruction of the direction and the energy of the muon track. In order to reach a muon angular resolution of $0.1^{\circ}$, it was shown [2] that the pulse from a single photon in the PMT must be measured with a timing precision of less than 2 ns RMS [2] and with a charge estimate in a dynamic range up to 100 photoelectrons (pe) [2,3]. Radioactive ${ }^{40} \mathrm{~K}$ decays and bioluminescence produce a continuous optical background in sea water. Simulations and measurements indicate that singles rates of $100 \mathrm{~Hz}$ per $\mathrm{cm}^{2}$ of photocathode [2] are expected for a threshold of $30 \%$ of the average one photoelectron signal which is the main signal seen by the PMTs.

An original analogue-to-digital data processing ASIC was developed based on the time over threshold (TOT) technique in order to read out the PMT signal with the following constraints: a high counting rate environment, good performance on single photoelectron but also the ability to deal with continuous background level due to random signals from the deep sea [4]. To analyze the data coming out of the chip, a general method for charge and time reconstruction based on the TOT has been implemented and fully tested with real data from the ASIC.

In the next section the principle of data processing is described followed by the technical details and performances of the ASIC. Then the algorithm development and data analysis for PMT signal 
processing are presented. The last section is dedicated to the ASIC performances with a PMT.

\section{Principle of data processing}

\subsection{Double sampling in time and in amplitude}

The principle of the TOT data processing used in the ASIC is illustrated in Fig. 1. The input analogue voltage is compared with several thresholds which can all be set independently. The comparator outputs are sampled by a fast virtual clock and saved in a digital memory. Thus the image of a PMT pulse is a digital waveform where the amplitude precision depends on the number of thresholds crossed by the signal. The time precision depends
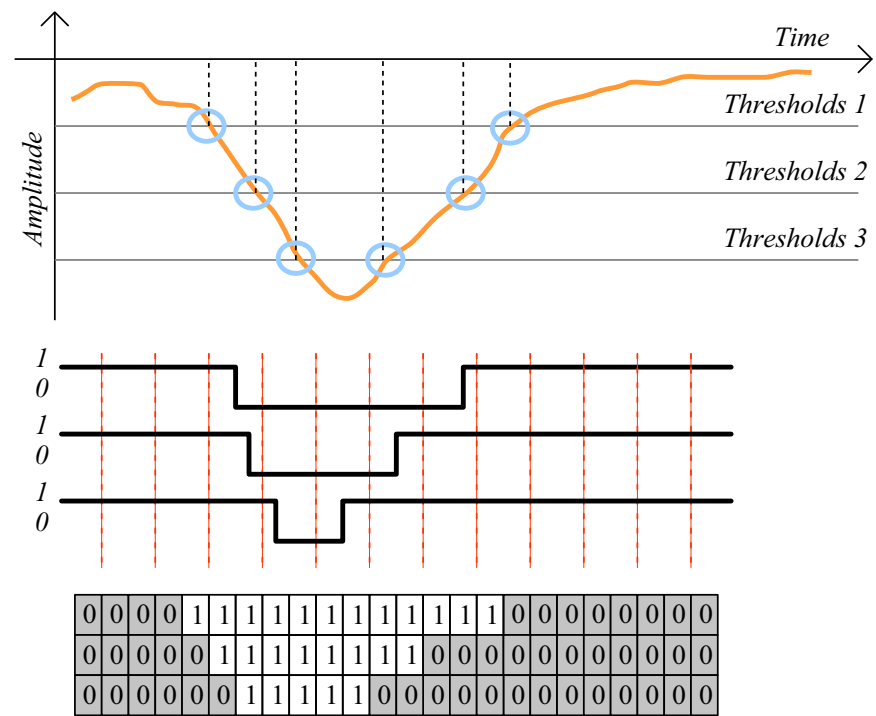

Fig. 1. Principle of SCOTT ASIC data processing. The analogue signal is sampled twice, first by discriminators, second, in time by a clocked system. The outputs are saved as a waveform. on the sampling rate. The threshold values can be non-linearly distributed in order to focus on voltage ranges of interest. The output data rate can be controlled by the number of thresholds used and by the sampling frequency.

\subsection{ASIC architecture}

The SCOTT ASICs were built and tested as a hardware implementation of this original data processing. The AMS BiCmos $0.35 \mu \mathrm{m}$ process technology was chosen for its low offset and fast switching capabilities. The full functionality prototype is composed of 16 independent channels each divided into three sub-circuits: a comparator stage followed by a circular sampling memory and a "first in first out" (FIFO) digital memory (Fig. 2). The independent channels let the ASIC be used either like a flash ADC or like a TDC, depending on the chosen input configuration. Unlike the ARS ASIC previously produced for the specific PMT readout scheme of the operational ANTARES neutrino telescope [5], the versatility of configurations permitted by the free set of thresholds let the ASIC match the time and charge requirements for the two options of light sensors proposed for KM3NeT with a common electronic readout. The two possible options are one 10-in. PMT [3] or sixteen 3-in. PMTs [3]. Indeed in the case of the 10-in. PMT option, the PMT is connected to several discriminators in order to increase the accuracy of charge reconstruction. The maximum resolution as a classical flash $\mathrm{ADC}$ is obtained when the same analogue signal is sent to the 16 channels and the thresholds are linearly distributed: in this configuration the ASIC behaves as a 4-bit ADC running at $800 \mathrm{MHz}$. In addition to a conventional ADC, thresholds can be set only in regions of interest in order to decrease the number of channels needed per source (Fig. 3a). In the second option, sixteen 3-in. PMTs are connected each to one channel: in this configuration the ASIC behaves as a 16-channel TDC with a 1.25 ns time step (Fig. 3b, the description of the sampler is detailed Section 3.3) for both signal edges, well suited for TOT measurement. In addition to a conventional TDC, the architecture of the ASIC concatenates the discriminator digital outputs into the FIFO, giving a trigger correlation between the samples if signals appear in the same time slice of $20 \mathrm{~ns}$.

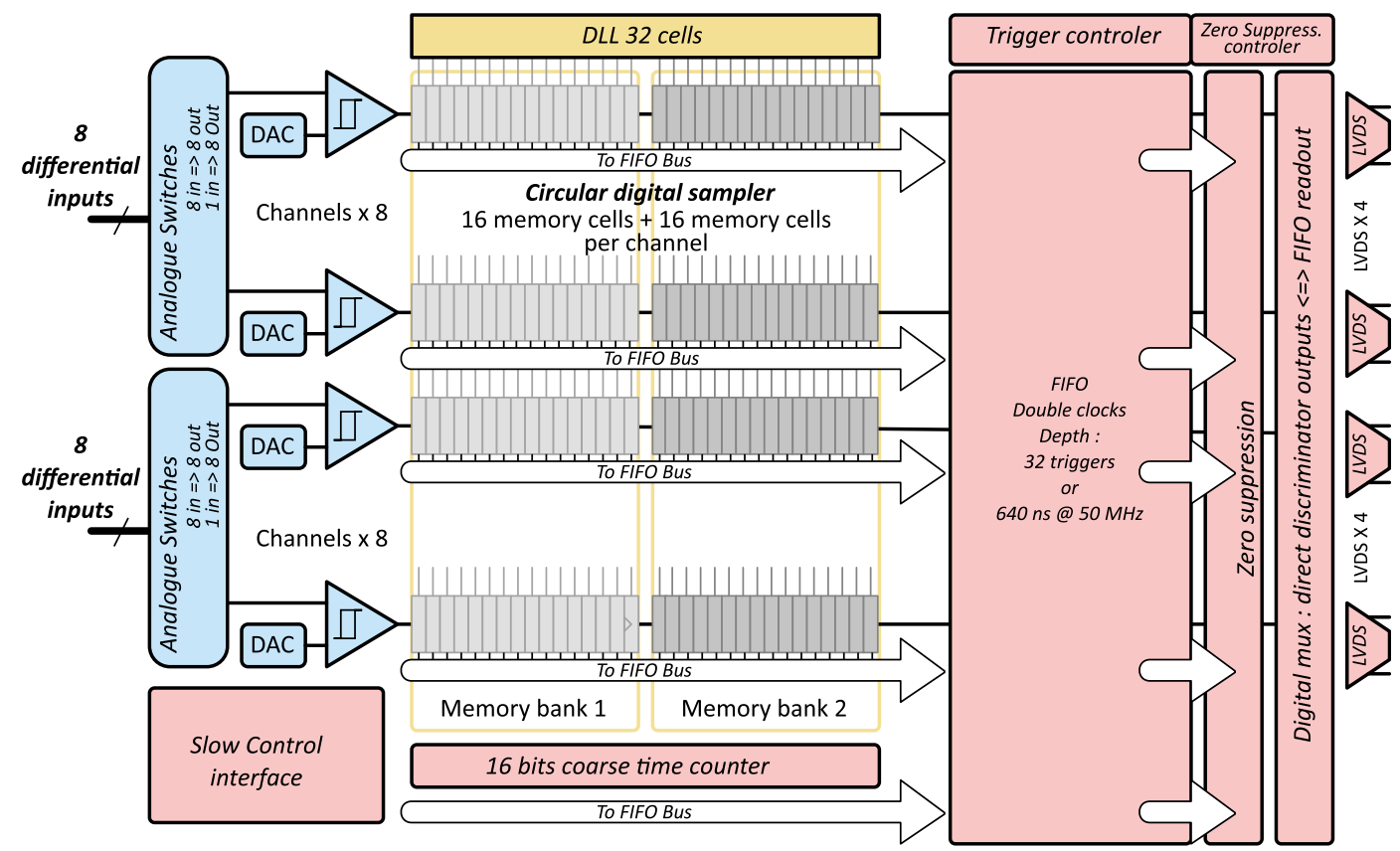

Fig. 2. 16-channel architecture of the SCOTT ASIC. 
a

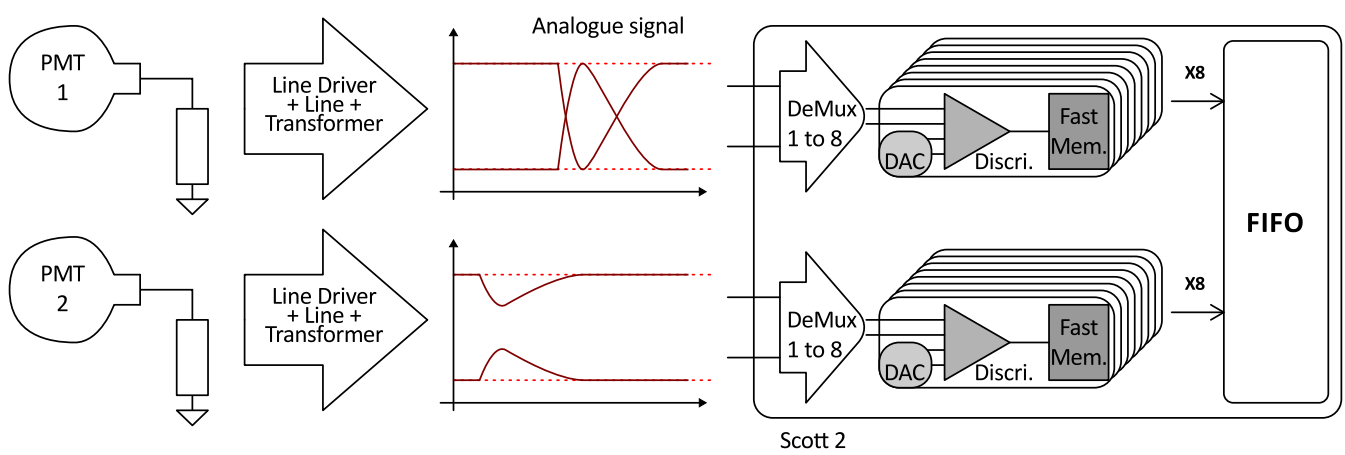

b

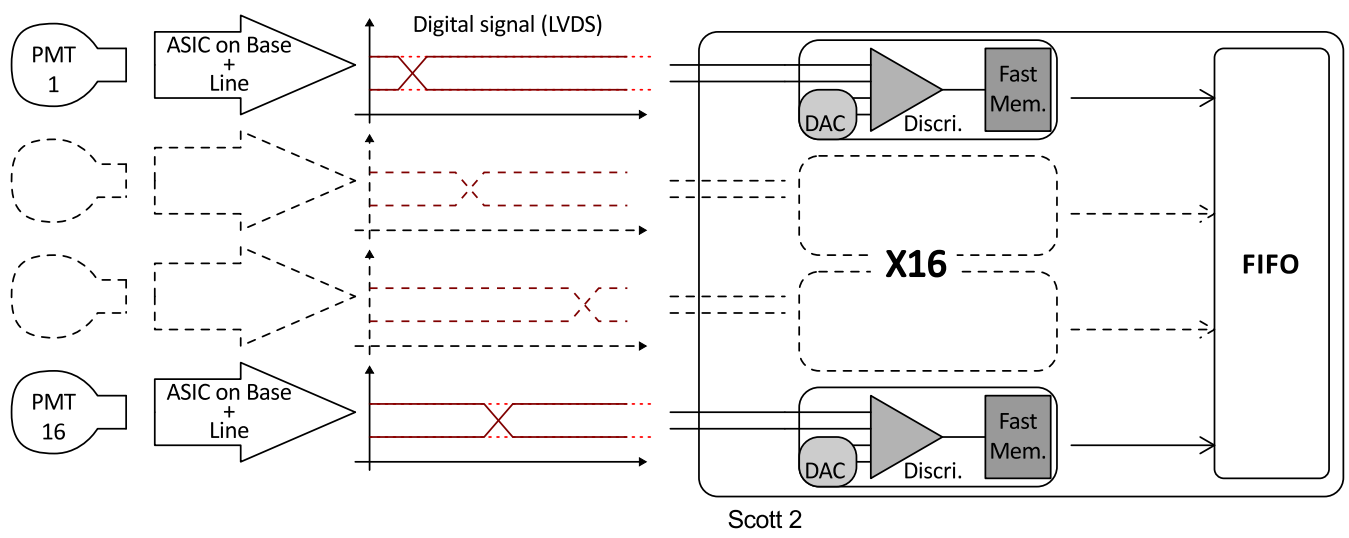

Fig. 3. (a) Schematic of two 10 -in. PMTs connected to the SCOTT ASIC. (b) Schematic of $16 \times 3$-in. PMTs connected to the SCOTT ASIC.

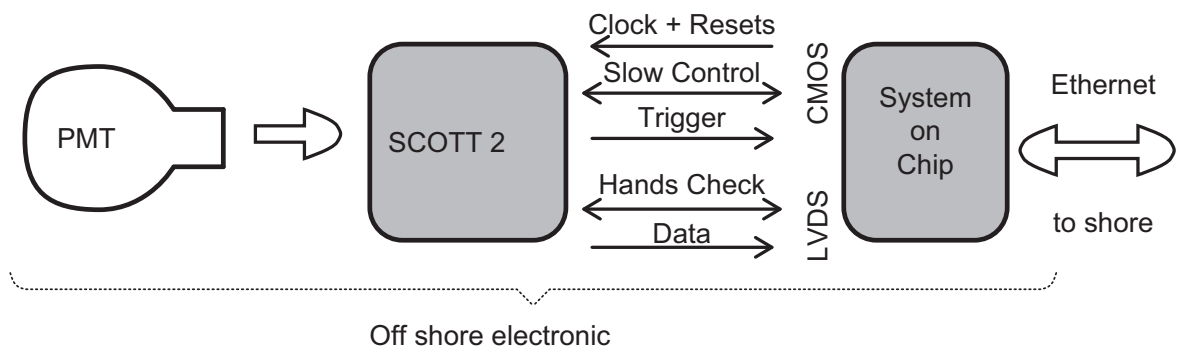

Fig. 4. Readout architecture connecting the SCOTT ASIC to the shore.

\section{SCOTT ASIC main functionalities and intrinsic performance}

\subsection{Experimental setup}

Prototypes of the ASIC have been fully tested in the laboratory with hardware compatible with the readout electronics foreseen for the KM3NeT experiment (Fig. 4). Two test benches have been developed: the first one aimed at validating the ASIC in a controlled environment (i.e. the 16 inputs of the ASIC can be individually addressed and all the analogue reference voltages freely set) whereas the second used the ANTARES acquisition system - inherited from ANTARES hardware to a System On Chip (SoC) - in order to validate the behavior of the ASIC on a board compatible with the embedded constraints and with a real data acquisition system.

\subsection{Analogue inputs}

Each channel is independent with a dedicated input in order to treat signals from different sources. Nevertheless, an on-chip switch network is implemented to route the internal analogue signal up to eight discriminators. The complete analogue chain is differential in order to remove parasites induced by the fast sampling clock signal of the digital part. It is composed of a discriminator for which the threshold is set by an internal 10-bit resistor string digital to analogue converter (DAC). There is one DAC per channel and all DACs are individually programmed through a slow control interface compatible with the serial to parallel interface (SPI) protocol. The differential DAC is based on two single ended 9 bit DACs [6] driven by a digital decoder in order to produce the 1023 voltage steps. The ladder architecture of the DAC ensures the output monotonicity and exhibits a $10 \mathrm{bit}$ resolution with a maximal dynamic range of $2.9 \mathrm{~V}$. The absolute values of DAC differential and integral non linearity are both less $0.5 \mathrm{LSB}(\mathrm{LSB}=\mathrm{UDAC} \sim 2.85 \mathrm{mV})$.

The discriminator is a fully differential structure with four stages: the input stage is done with two differential pairs followed by two gain stages and terminated by an asynchronous latch (Fig. 5a). The chain exhibits a total gain of $41 \mathrm{~dB}$ before the latch and a measured input noise of $700 \mu \mathrm{V}$ RMS. Since up to 16 discriminators could be connected to a common source, the input current of each discriminator should be kept as low as possible. Indeed, depending of the 
a

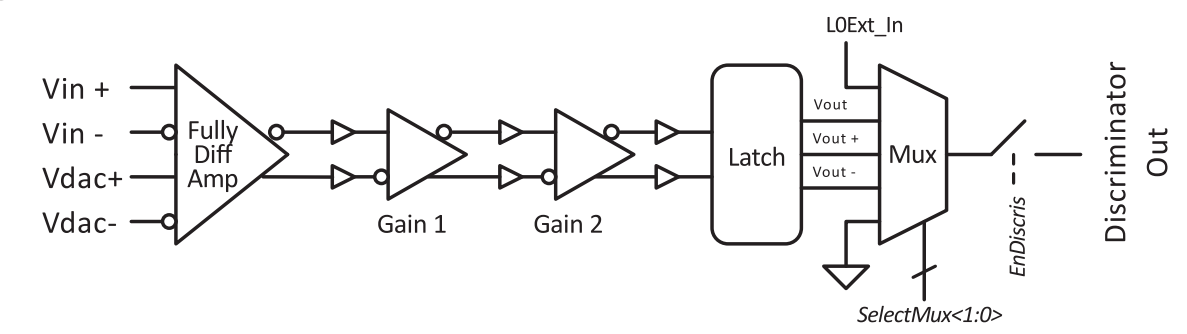

b

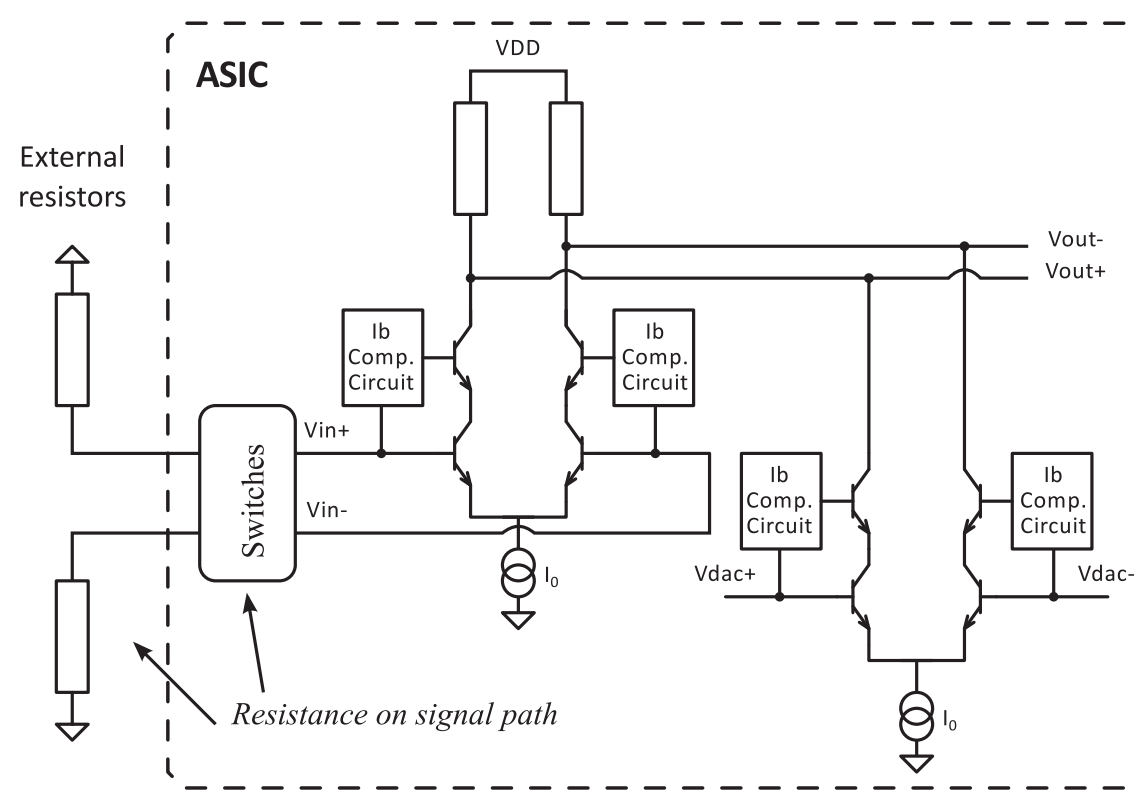

Fig. 5. (a) Block diagram of discriminators. (b) Schematic of the discriminator input stage.

state of the discriminator (output at one or zero), the input bipolar transistors will need different base currents. This current variation into the serial input resistor leads to crosstalk between channels. Thus, the input stage is done with bipolar transistors and an input current compensation circuit which leads to largest measured input current of $85 \mathrm{nA}$ (Fig. 5b). Into $100 \Omega$, the crosstalk between the 16 channels is kept below $16 \times 85 \times 10^{-9}=1.36 \mathrm{mV}$ which is less than 0.5 LSB. The offsets between channels have been measured higher in the last prototype than in previous ones which was below $1 \mathrm{mV}$ RMS as expected from simulation. The origin of the offsets has not been understood and external causes have not been rejected yet. In practice, the offsets from discriminators are easily compensated by calibration.

The overall bandwidth of the system depends on the inputs' configuration and especially from the switch network settings due to the resistance added to the signal path. For the worst case scenario, when the switch network connects eight channels to one input, the bandwidth and timing performances are summarized in Table 1. The most favorable configuration is obtained when one channel is connected to one input: in these conditions the bandwidth is expected to reach $350 \mathrm{MHz}$.

\subsection{Digital sampling and readout}

For tests or special operations, the digital signals from discriminators can bypass the digital sampler and can be sent directly to low voltage differential signal (LVDS) outputs with the possibility to power down the rest of the digital part. In nominal operations, the outputs of the discriminators are sent to a circular digital memory.
Table 1

Bandwidth and timing performances of the 16 discriminators for the worst case scenario: eight channels are connected to 1 input through the internal switch network and the overdrive moved from $100 \mathrm{mV}$ to $1 \mathrm{mV}$.

\begin{tabular}{llll}
\hline & Bandwidth & Max. $\Delta$ Skew & Max. $\Delta$ Delay \\
\hline Measure & 180 & $<500$ & $<700$ \\
Simulation & 210 & & $<650$ \\
Unit & MHz & ps & ps \\
\hline
\end{tabular}

The 16 discriminator outputs are simultaneously sampled into a circular memory. The circular memory is divided into two subsets of 16 cells working alternately in order to remove the dead-time during the backup of data in the FIFO. The delay between samples are guaranteed thanks to a delay locked loop (DLL) which divides by 16 the ASIC main clock period and provides 16 virtual delayed clocks. With a $50 \mathrm{MHz}$ main clock frequency the DLL produces a time step of $1.25 \mathrm{~ns}$ equivalent to a sampling clock running at $800 \mathrm{MHz}$. The ASIC is auto-triggered with the possibility to set each channel as a trigger. The trigger is directly produced from the data saved in the circular memory to guarantee a perfect synchronism between the circular memory and the FIFO. The derandomization of the incoming signal is done inside the ASIC within the FIFO: only triggered events are saved and tagged with a 16 bit time-stamp provided by a counter. The final dynamic range achievable by combining the counter and the DLL is 20 bits at the virtual clock frequency (i.e. $800 \mathrm{MHz}$ in the nominal case).

When at least one trigger is detected, the newly written 16 cells of one subset of the circular memory are stored into the FIFO. The 16 channels are saved regardless of the presence of signal. Thus 
a trigger produces the storage of a time slice of 16 synchronous digital data per 16 channels namely 256 bits. At nominal frequency, the time slice corresponds to $16 \times 1.25 \times 10^{-9}=20 \mathrm{~ns}$. The FIFO can save up to 32 consecutive time slices equivalent to a continuous 640 ns waveform in nominal conditions. The FIFO has a "double ports" architecture and accepts a readout clock different from the writing clock. Channels which have not seen a signal during a time slice are not sent to the readout by "on the flight" zero-suppression. All tests have been performed with a readout clock equal to the ASIC main clock. Fig. 6 presents the readout performance expected in these conditions as a function of the number of channels assigned to a common analogue input and the Poisson distributed input rate seen by a 10 -in. PMT.

The loss of input data is due to the Poisson distributed process of the incoming photons and the limited size of the FIFO. It can be seen from Fig. 6 that the ASIC can run with an input rate at $1 \mathrm{MHz}$, with three thresholds placed in the average single-photon response amplitude and with a loss of input data around $1 \%$.

\subsection{Results from raw data}

The data delivered by the ASIC are time slices of 20 ns. The Fig. 7a shows an example of a reconstructed waveform with an input pulse from a HP 8111A pattern generator. Abutted together

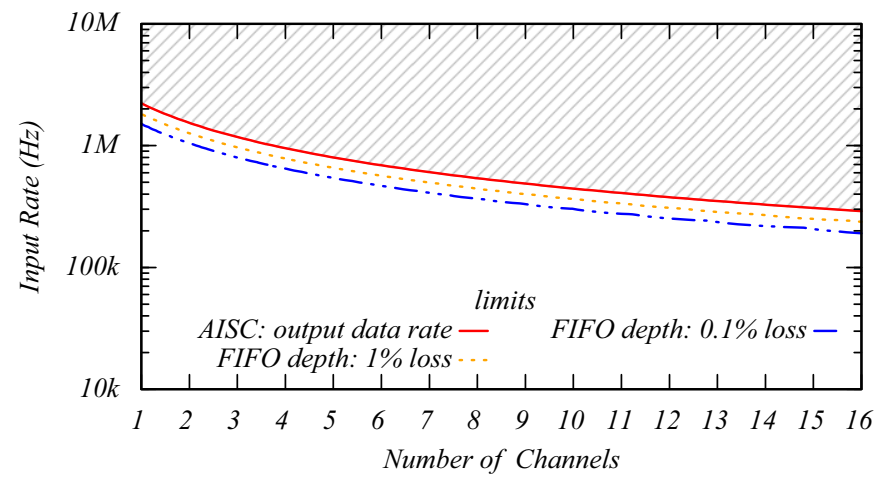

Fig. 6. Readout limits depending on two parameters: the Poisson distributed input rate seen by a 10 -in. PMT and the number of channels assigned to a common analogue input. they form a digital waveform where the difference of times between the columns is $1.25 \mathrm{~ns}$ and each line represents one channel thus one threshold level. In this example, the thresholds are equally distributed with a difference between consecutive thresholds of $2 \times$ UDAC $\sim 5.7 \mathrm{mV}$. The raw data have been averaged with about 100 measurements and compared with the same pulse digitized by a fast oscilloscope. The Fig. 7b shows the good agreement between the two types of measurement.

The pulse reconstruction allows the evaluation of the intrinsic timing performance of the ASIC. The reference pulses from the generator were a triangle signal (120 ns total length) with a $150 \mathrm{mV}$ amplitude. Six thresholds were placed irregularly over the dynamic range. The mean value is removed in order to keep only the time variance. The RMS time resolution of SCOTT is $0.8 \mathrm{~ns}$ in this condition. This time resolution includes the intrinsic timing performance of the ASIC and the contribution from the threshold variations ( $0.7 \mathrm{mV}$ RMS) combined with the relatively slow slopes from the input signal. The effect of the input noise is attenuated with the fastest signals and thus the timing resolution should be better with PMT signals exhibiting faster slopes than the triangle signal used.

\section{Time and charge reconstruction methodology}

As described previously, the SCOTT ASIC acts as a sampling device in time and in amplitude. The amplitude is given by the choice of the thresholds and the time information corresponds to their crossing point with the input signal. In case of the 10-in. PMT option, the elements to be determined are the number of thresholds, their amplitude and the method allowing the pulse time and charge to be obtained with resolutions respecting the KM3NeT [2] requirements. A set of PMT pulses were digitized using an oscilloscope. A mathematical representation of the pulse allowed the number and positions of the thresholds to be found, as well as the peak time and charge of the pulse. For this, we use a function to fit the pulses. A simpler estimation of the pulse time and charge was found from the duration above the threshold.

\subsection{Data used for the preliminary analysis}

A set of $\sim 10^{4}$ pulses were recorded with ANTARES optical module [7], housing a Hamamatsu R7081-20 PMT, placed in a a

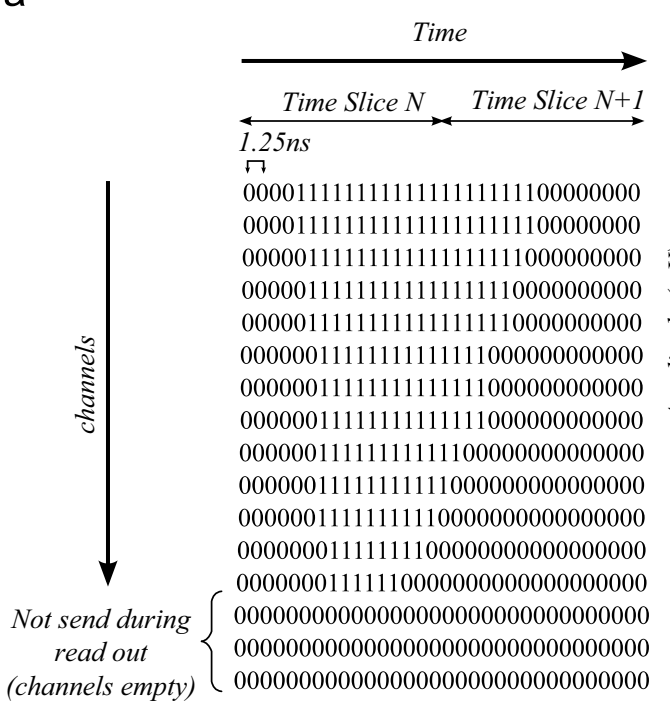

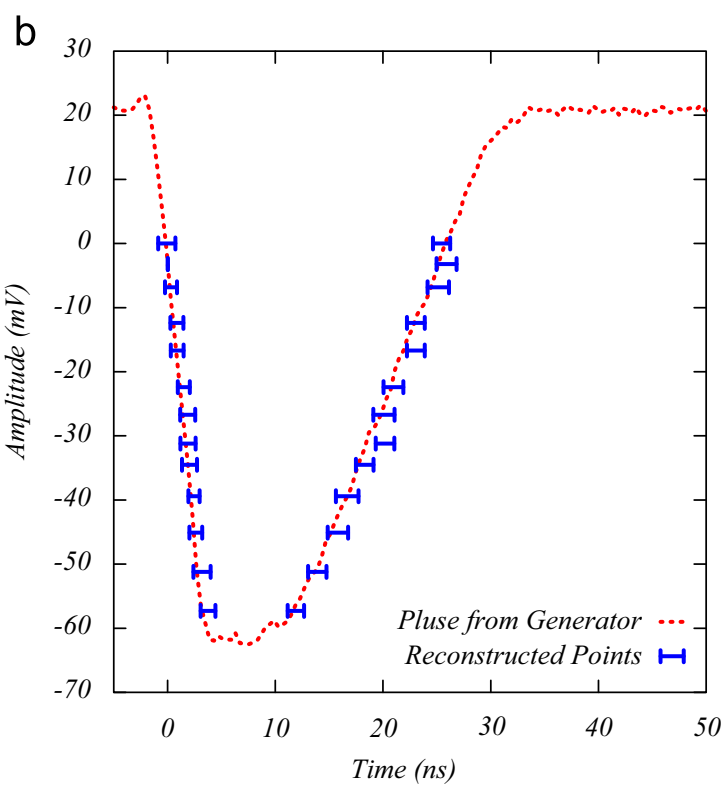

Fig. 7. (a) Digital data output: Ascii Art reconstruction from two time slices. (b) Reconstruction of pulse from generator: average of 100 waveforms. 
black box and illuminated with a LED. Using the PMT dark noise, the one photoelectron amplitude level was found at $40 \mathrm{mV}$ corresponding to a charge of $8 \mathrm{pC}$ or an equivalent gain of $5 \times 10^{7}$. Varying the LED power enabled pulse amplitudes from $40 \mathrm{mV}$ to $3 \mathrm{~V}$ to be registered using an oscilloscope with a $2.5 \mathrm{GHz}$ sampling rate.

\subsection{Determination of the thresholds}

The shape of a PMT pulse exhibits a fast rising slope followed by a slower descending curve. It can be parametrized by the following function:

$a(t)=A\left(t-t_{0}\right)^{\alpha} e^{-\beta\left(t-t_{0}\right)}, \quad t>t_{0}$.

The maximum amplitude $(\hat{A})$ is at $\hat{t}=\alpha / \beta+t_{0}$. The rise time is defined as $t_{R}=\hat{t}-t_{0}$. Using these variables, the function can be rewritten

$a(t)=\hat{A}\left(\frac{t-\hat{t}}{t_{R}}+1\right)^{\alpha} e^{\left(-(t-\hat{t}) / t_{R}\right) \alpha} \times H\left(\hat{t}-t_{R}\right)$

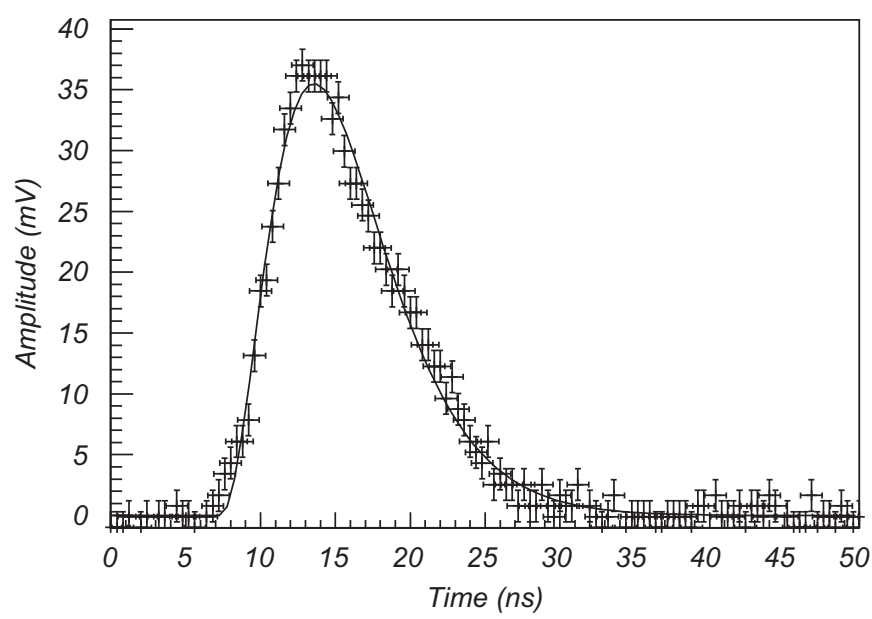

Fig. 8. Example of a 10 in. PMT pulse fitted with the Eq. (2).

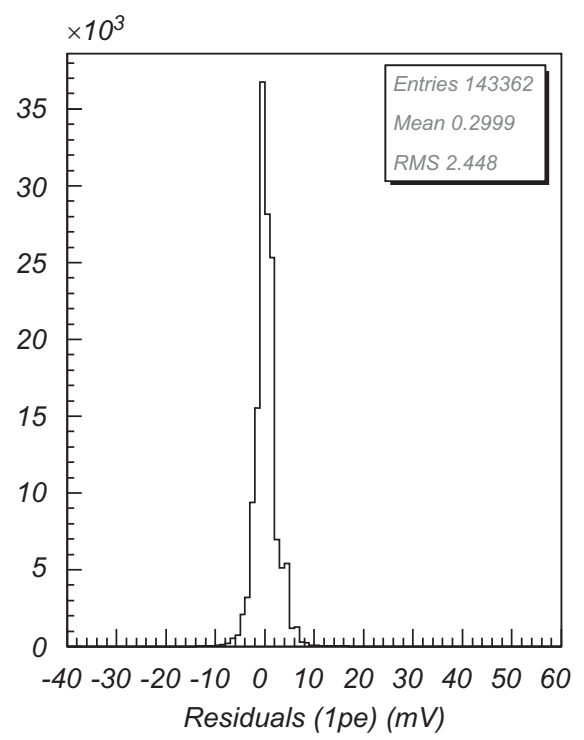

where $H$ is the Heaviside function. An example of a pulse fitted with Eq. (2) is presented in Fig. 8. The residuals of the fit for 1 pe and 25 pe are shown in Fig. 9.

This parameterization is used to determine the level and number of thresholds suited to retrieve the pulse function. The pulse time is chosen to be the peak time $(\hat{t})$ and the charge is simply the integral of the function over $50 \mathrm{~ns}$. The pulse times and charges from the fitting of Eq. (2) to the set of digitized pulses are taken as reference values. The number of points of these digitized pulses is then reduced following the SCOTT concept of operation: for a given number and level of thresholds, only the crossing points at the thresholds are kept. These points are then used to fit the same parameterization of Eq. (2) and their pulse times and charges are compared to the reference ones. By varying the number and level of thresholds, an optimized solution is obtained which minimizes the number of thresholds and fulfills the KM3NeT requirements.

With the five thresholds given in Table 2, the precision in the determination of the pulse time is better than $1 \mathrm{~ns}$ RMS up to 50 pe and the error on the charge is less than $20 \%$ up to 60 pe.

\subsection{Time over threshold estimate}

In a large detector as planned for KM3NeT, the implementation of the fit of each PMT pulse with Eq. (2) is not adapted. For a faster implementation of the time and charge estimation, a relation with the time over threshold is investigated.

The relation with the peak time $\hat{t}$ is derived from Eq. (2). Defining $t_{1}$ and $t_{2}$ the time at which the pulse crosses the threshold at amplitude $a_{n}$. The time over threshold is $\Delta t=t_{2}-t_{1}$ and $a_{n}=a\left(t_{1}\right)=a\left(t_{2}\right)$. The peak time $\hat{t}$ can be written as a function of $\Delta t$

$$
\hat{t}-t_{1}=\left(t_{2}-t_{0}\right) \times \frac{1}{X}\left(1-(1+X) e^{-X}\right)
$$

Table 2

Amplitude of the five thresholds needed to reconstruct the PMT pulse's charge and time for the KM3NeT requirements.

\begin{tabular}{llllll}
\hline Threshold value (pe) & $1 / 3$ & $2 / 3$ & 1 & 3 & 8 \\
\hline
\end{tabular}

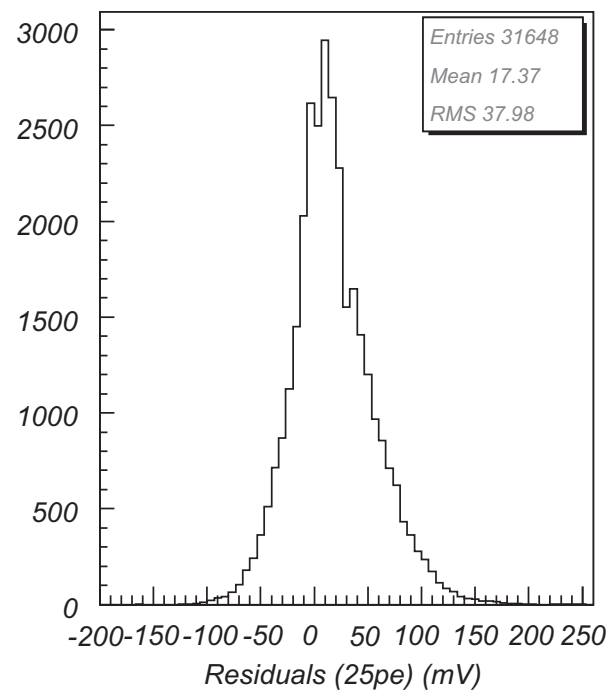

Fig. 9. Residuals of the fit using Eq. (2) on pulses recorded as detailed in Section 4.1 , for LED generated pulses at 1 pe and 25 pe amplitudes ( 1 pe $=40 \mathrm{mV}$ ). 
where

$X=t_{R} \Delta t$

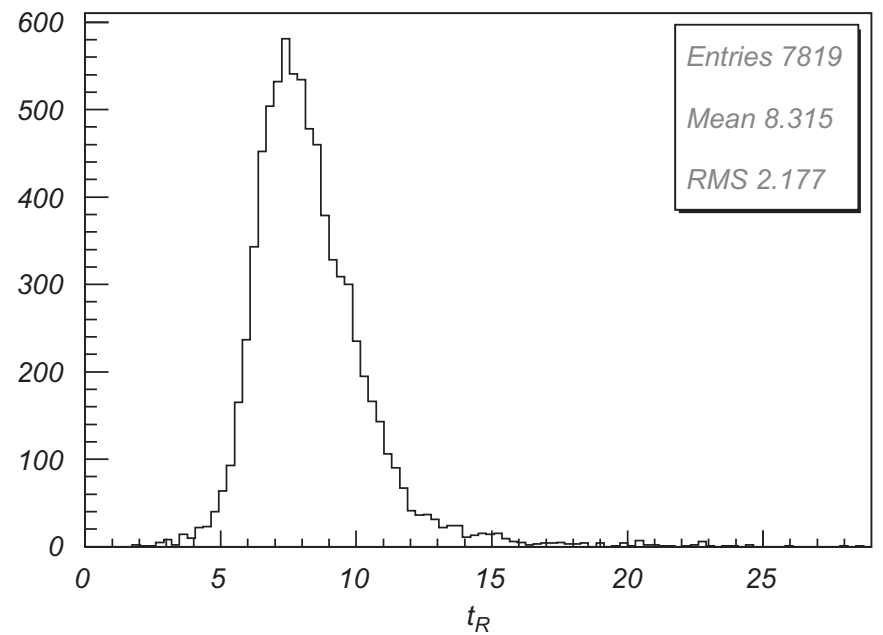

Fig. 10. Distribution of the parameter $t_{R}$ in Eq. (2), from the fit of pulses with amplitudes up to 50 pe.

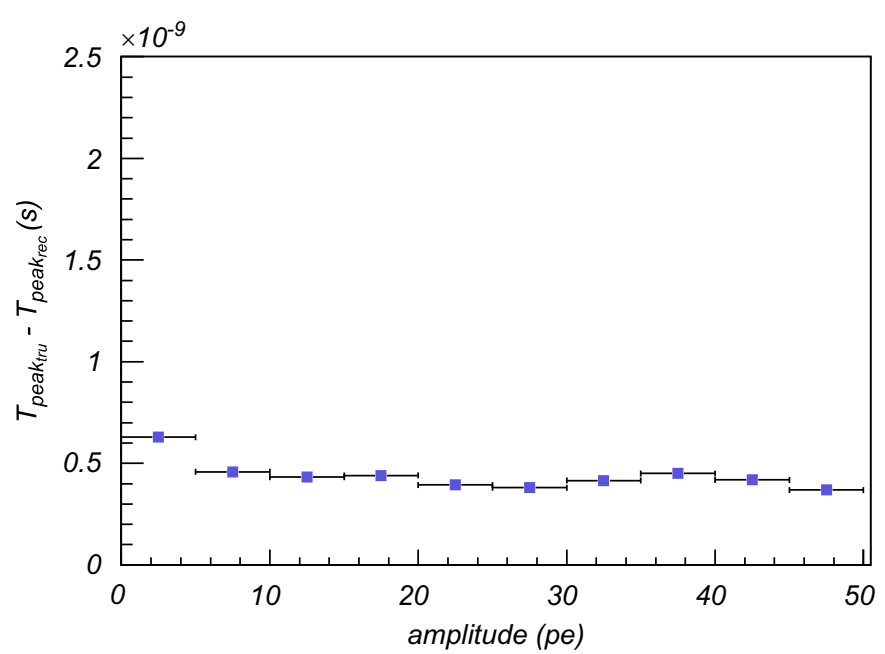

Fig. 11. Contribution of the time over threshold method to the time resolution (RMS per bin of 5 pe), as a function of the amplitude of pulses.

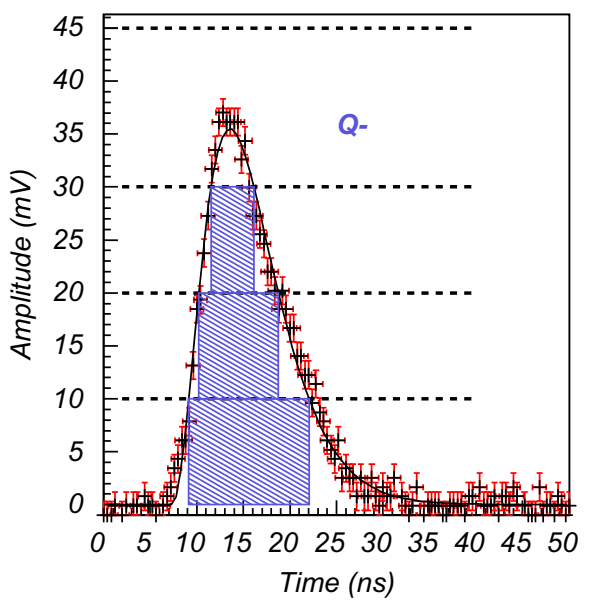

This relation is valid for any threshold, hence only one parameter, $t_{R}$, is needed and can be obtained from the fit with Eq. (2) over a "training" sample of PMT pulses. The distribution of $t_{R}$ for pulses produced with amplitudes up to 50 pe is shown in Fig. 10. The 2 ns RMS of $t_{R}$ is strongly linked to the time precision found with this method. The RMS resolution of $\hat{t}$ using all the 5 thresholds in Table 2 is $0.7 \mathrm{~ns}$. A better estimate is found using only the highest threshold crossed as can be see in Fig. 11 showing the RMS time resolution as a function of the pulse amplitude. The contribution of the method to the time resolution is of the order of $0.5 \mathrm{~ns}$. The peak time has the advantage of being insensitive to the walk effect, which appears as a consequence of the charge variation compared to the time measured directly at the crossing threshold.

The pulse integral - and so the charge - can be related to the TOT using the rectangle area under the thresholds. As the thresholds are not linearly distributed, an estimate of the charge is constructed with a combination of two areas: the sum of the rectangle areas above the thresholds $\left(Q_{+}\right)$and the sum of the rectangle areas below the thresholds $\left(Q_{-}\right)$. Thus the charge is written

$Q_{\text {tot }}=c_{Q} \times\left(Q_{+}+Q_{-}\right)$

$Q_{-}=a_{0} \Delta t_{0}+\sum_{i=1}^{N}\left(a_{i}-a_{i-1}\right) \Delta t_{i}$

$Q_{+}=\sum_{i=1}^{N} a_{i} \Delta t_{i-1}+ \begin{cases}a_{N+1} \Delta t_{N}, & N<N_{k} \\ a_{N_{k}} \Delta t_{N_{k}}, & N=N_{k}\end{cases}$

where $N$ is the last threshold reached by the pulse, and $N_{k}$ is the number of defined thresholds, i.e. $N_{k}=5$. An illustration of $Q_{+}$and $Q_{-}$is shown in Fig. 12. $c_{Q}$ is a constant factor for pulses with amplitudes up to the last defined threshold, as long as the time over the last threshold is shorter than $10 \mathrm{~ns}\left(\Delta t_{N_{k}}<10\right)$. For higher charges, the total charge $Q_{t o t}$ is no longer proportional to the estimate $Q_{+}+Q_{-}$, but also depends on the time over the last threshold $\left(\Delta t_{N_{k}}\right)$. The factor $c_{Q}$ is then taken as a $1 \mathrm{~d}$-polynom of $\Delta t_{N_{k}}$, with its two parameters also determined from the charge estimated from the fitted pulses.

Using this estimator, the error on the charge is better than $15 \%$ up to 40 pe and degraded to $20 \%$ up to 60 pe, as it can be seen on Fig. 13.

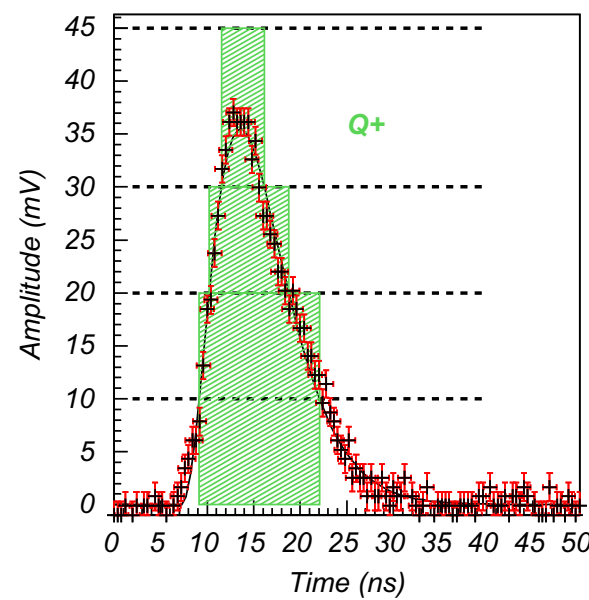

Fig. 12. Illustrated example of the charge determination using a combination of the rectangle areas above $\left(Q_{+}\right)$and below $\left(Q_{-}\right)$the thresholds. 


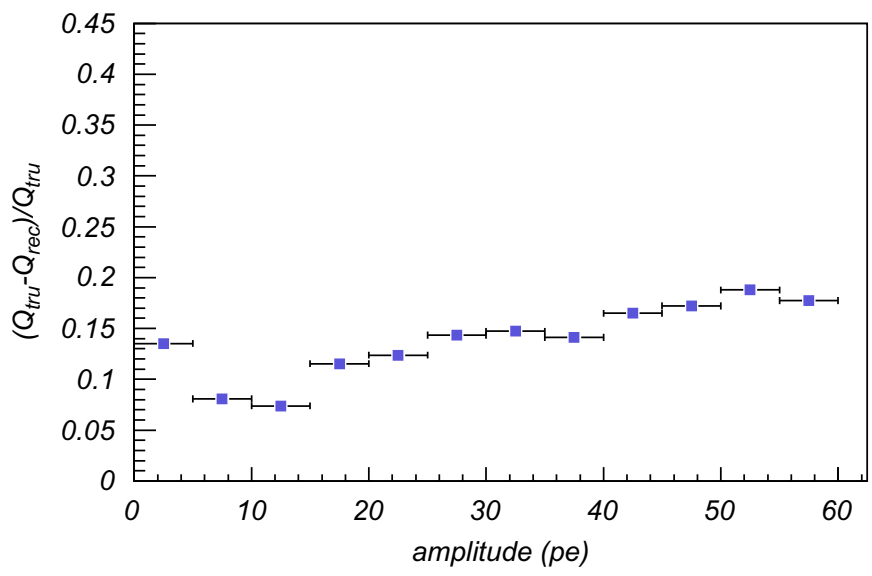

Fig. 13. Reduced error on the reconstructed charge using the TOT method $\left(Q_{+}\right.$and $Q_{-}$) presented as a function of the pulse amplitude.

Table 3

Mean and RMS (in brackets) of the charge distributions for the pulses registered with the oscilloscope, with the SCOTT ASIC using the function in Eq. (2) and with the TOT method.

\begin{tabular}{lll}
\hline Measurement method & $1 \mathrm{pe}$ & $11 \mathrm{pe}$ \\
\hline Scope (Ref.) & $5.3 \mathrm{pC}(1.9 \mathrm{pC})$ & $66 \mathrm{pC}(21 \mathrm{pC})$ \\
SCOTT Eq. (2) & $5.1 \mathrm{pC}(1.9 \mathrm{pC})$ & $70 \mathrm{pC}(22 \mathrm{pC})$ \\
SCOTT TOT & $5.2 \mathrm{pC}(2.1 \mathrm{pC})$ & $63 \mathrm{pC}(20 \mathrm{pC})$ \\
\hline
\end{tabular}

\section{Performance of the SCOTT ASIC reading a 10-in. PMT}

The two methods described in Section 4 are applied to the SCOTT ASIC connected to a PMT. In what follows, the data taking is described and the performances are given.

\subsection{Data taking}

An ANTARES 10-in. PMT is put in the black box and illuminated with the LED reproducing amplitudes of 1 pe or 11 pe. The SCOTT ASIC records the PMT pulses with the five thresholds defined in the previous section (Table 2). A dedicated channel registers the trigger signal from the LED, used for coincidences. Data are also taken with the same set up with an oscilloscope digitizing the pulse for further reference. The gain of the PMT is set to $3 \times 10^{7}$ which produces a 1 pe amplitude distribution centered at $53 \mathrm{mV}(5 \mathrm{pC})$.

\subsection{Charge determination}

Table 3 shows the results in terms of mean and RMS values of the charge, for the measurements with the oscilloscope and the ASIC. The data from the ASIC were reconstructed with the two methods: the fit with Eq. (2) and the time over thresholds estimate (TOT method). As an example, Fig. 14 shows the distribution of the reconstructed charge with the TOT method for pulses at 1 pe. Either of these two methods gives an uncertainty in the determination of the charge which is below the intrinsic PMT charge resolution (around 30\%).

\subsection{Time determination}

In order to check that the time resolution in a realistic experimental setup remains below the requirements ( $2 \mathrm{~ns})$, the time difference between the pulse peak time in SCOTT and the time of LED trigger were measured at 1 pe and 11 pe. Fig. 15 shows this time difference when the peak time is obtained from

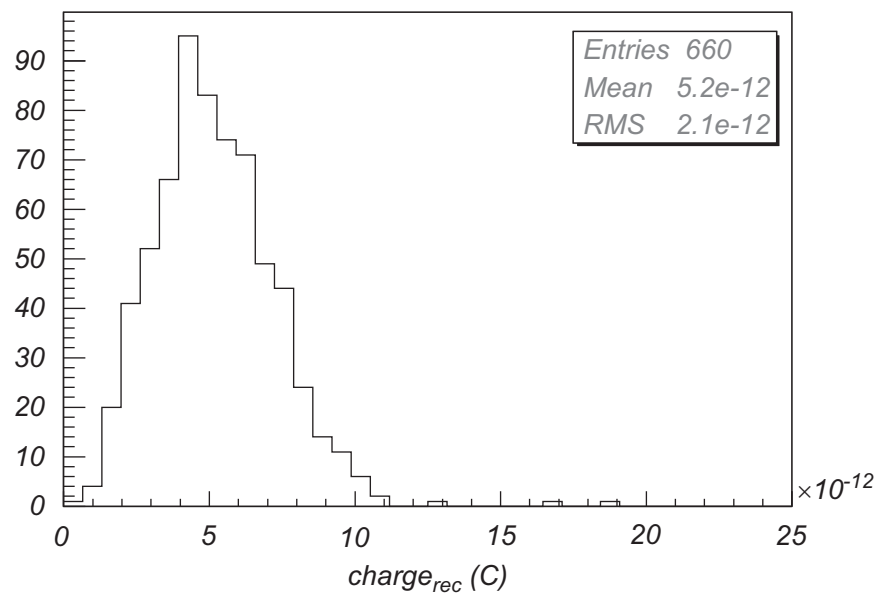

Fig. 14. Charge estimated from SCOTT, with time over thresholds for PMT pulses at 1 pe.
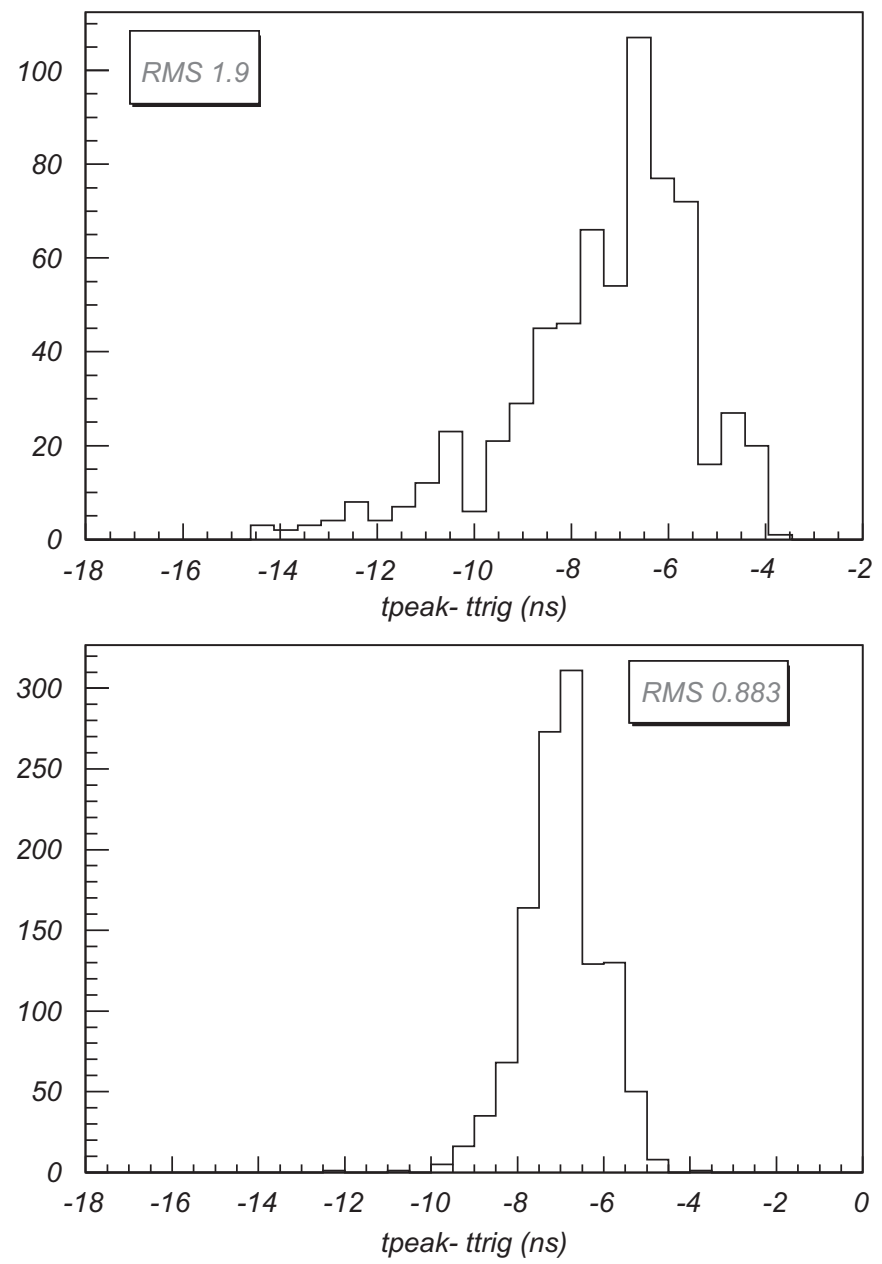

Fig. 15. Distribution of the difference between the peak time and the trigger time, with SCOTT and the time over threshold estimate, above for 1 pe pulses, below for 11 pe pulses.

the TOT method. The transit time spread (TTS) of ANTARES optical module is $1.3 \mathrm{~ns}$ at 1 pe [7]. This TTS becomes much smaller for higher amplitudes. The time resolution that includes both the SCOTT intrinsic time resolution and the TTS is 1.9 and 0.9 ns for 1 and 11 pe respectively. Similar results are obtained with the fit 
method. The overall timing resolution is therefore below 2 ns at any charge value.

\section{Conclusion}

A new analogue-to-digital converter ASIC has been designed for PMT signal readout based on the multi-time-over-threshold technique. It combines both best features from an ADC and a TDC with extra functionalities as auto-triggering and derandomization of incoming signals. The intrinsic performances of the ASIC are a low input referred noise ( $0.7 \mathrm{mV}$ RMS) over a large 10-bit dynamic range giving the possibility to set the thresholds only at regions of interest. The time accuracy measured is better than $0.8 \mathrm{~ns}$ RMS. The flexible architecture of the ASIC makes it suitable either to multiple 3-in. or to single 10-in. PMTs. With 10-in. PMT, it was found that the best time and charge performance is obtained with five thresholds between 0.3 and 8 pe. The charge can be estimated from the threshold information with a resolution (20\%) which is below the PMT intrinsic charge resolution. The information given by the last threshold allows a determination of the peak time, with a resolution of $0.5 \mathrm{~ns}$, which was checked to be independent of the charge up to 50 pe. With a 10-in. PMT having a transit time spread of $1.3 \mathrm{~ns}$, the overall timing resolution, including the intrinsic Scott resolution and the method uncertainties was found to be $1.9 \mathrm{~ns}$. Altogether, its versatile data processing makes the ASIC relevant for any application which requires time precision, derandomization and non-linear amplitude discrimination and coding.

\section{Acknowledgement}

The KM3NeT project is supported by the EU in FP6 under contract no. 011937 and FP7 under Grant no. 212525. The authors thank the NDIP organizers for their hospitality and a very well organized, inspiring conference.

\section{References}

[1] KM3NeT web site, 〈http://www.km3net.org/〉.

[2] The KM3NeT Consortium, Conceptual Design Report, 2008, ISBN 978-90-6488031-5.

[3] The KM3NeT Consortium, Technical Design Report, 2010, ISBN 978-90-6488033-9.

[4] J. Craig, et al., Nuclear Instruments and Methods in Physics Research Section A 602 (2009) 224-226 (Proceedings of the Third International Workshop on a VLVNT for the Mediterranean Sea).

[5] J.A. Aguilar, et al., Nuclear Instruments and Methods in Physics Research Section A 622 (2010) 59.

[6] C.-C. Chen, et al., A 10-Bit folded multi-LSB decided resistor string digital to analog converter, in: Proceedings of the International Symposium on ISPAC, 2006, pp. 123-126.

[7] P. Amram, et al., Nuclear Instruments and Methods in Physics Research Section A 484 (2002) 369. 